\title{
Double outlet right ventricle with L-malposition of great arteries, ventricular D-loop, and three fibrotic leaflets in a stenotic subpulmonary conus
}

\author{
A. Blancquaert, E. Defloor, L. Bossaert, M. Kunnen, M. De Maegd, and F. Derom \\ From the Departments of Paediatrics, Pathology, Cardiology, Radiology, and Surgery, University of Gent, \\ Belgium
}

A case of double outlet right ventricle, ventricular D-loop, L-malposition of the great arteries, and stenosis of the pulmonary outflow at valvar, supra-, and subvalvar level in a patient with situs solitus is described.

Fibrotic structures closely resembling semilunar valves turned upside down, with the three pockets looking upstream were found in the subpulmonary infundibulum. They lay parallel to the pulmonary valve and their commissures corresponded exactly with the commissures of this valve.

These fibrotic leaflets did not interfere with passage of the surgeon's finger or a dilator when the heart was empty, but may well have increased the infundibular stenosis during life.

The combination of a double outlet right ventricle with a D-loop ventricular position and L-malposition of the great arteries in an individual with situs solitus has only recently been described and only 7 cases have been reported (Paul, Van Praagh, and Van Praagh, 1968; Hallerman et al., 1970; Van Praagh et al., 1973). The valve-like structures found in the infundibulum, about $\mathrm{I} \mathrm{cm}$ below a normal pulmonary valve, in the case reported here, have not previously been reported. Their origin is uncertain, but they doubtlessly influenced the fatal outcome of the surgical intervention.

\section{Case report}

The only child of healthy parents, had a heart murmur at birth. She developed normally, but from the age of I year was noted to be dyspnoeic and cyanosed on exertion. These symptoms progressed slowly.

At the age of 7, an initial cardiac examination revealed a precyanotic child with some clubbing of fingers and toes. A thrill was felt and a stenotic $4 / 6$ systolic murmur was heard maximally at the second left and right intercostal spaces. The electrocardiogram showed right ventricular hypertrophy associated with a prominent $Q$ wave in the left praecordial leads. The chest $x$-ray revealed clear lung fields, right ventricular enlargement with a slightly concave upper right heart border, and a right-sided aortic arch. At catheterization (Table) the catheter passed from the right ventricle into the aorta, but no pulmonary artery was reached. Both ventricles and the aorta had high oxygen saturations and equal systolic pressures. The wedge pressure in the pulmonary vein was low. An injection of contrast medium in either ventricle filled a large aorta and showed very slight opacification of the pulmonary artery branches. The child was sent to Houston for a corrective operation for tetralogy of Fallot, but the surgeon (Dr. M. DeBakey) found a 'corrected transposition of the great vessels' and decided that the risk of an operation was too high at that moment.

She remained quite well up to the age of 15 years when she was admitted to hospital for vertigo and syncope with cyanosis and heart palpitations on exertion. The cyanosis had become more apparent at rest, but auscultation, electrocardiogram, and the chest $x$-ray had not much changed. The upper left heart border had become straighter. Electrocardiographic monitoring revealed periods of bradycardia with disappearance of the $P$ waves, nodal escape, and ventricular tachycardia. Recatheterization revealed only one new finding: an aortic oxygen saturation of 85 per cent (Table). Cineangiocardiography (Fig. I) showed a well-trabeculated right-sided ventricle giving rise to both great arteries in L-malposition. Both semilunar valves were at about the same horizontal level and there was no continuity of either of them with the mitral valve. An infundibulum was present under each semilunar valve, separated by a huge tear-shaped crista. The subpulmonary infundibulum showed conspicuous and localized narrowing in systole, but opened up well in diastole. Some stenosis of the valve and of the bifurcation of the pulmonary artery was 
TABLE Catheterization data

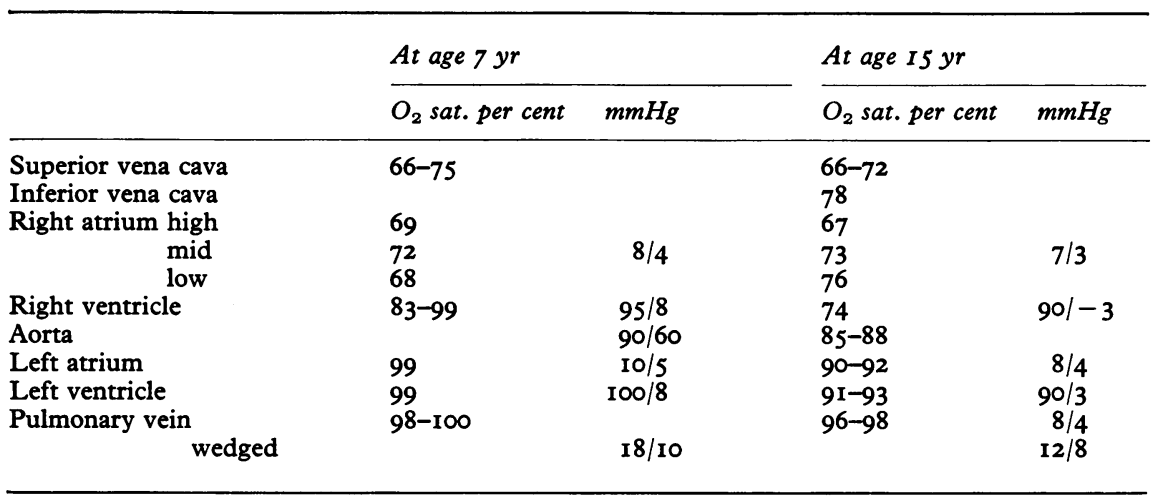

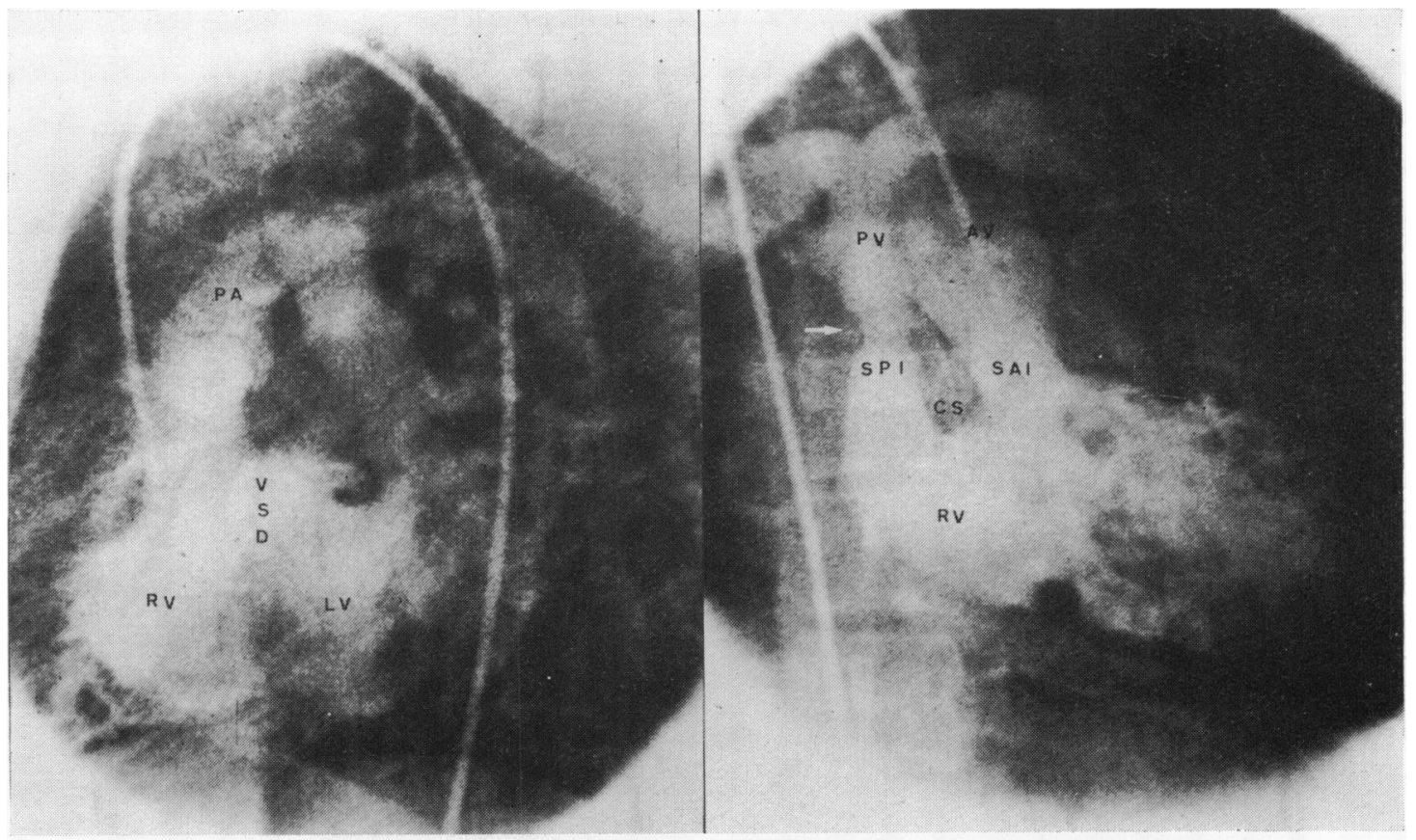

$a$

$b$

FIG. I Retrograde cineangiocardiography. The catheter enters the right ventricle from the aorta. (a) Lateral projection. Injection in the right ventricle $(R V)$ fills the pulmonary artery $(P A)$ and the left ventricle $(L V)$ via a ventricular septal defect $(V S D)$. The pulmonary valve is dome shaped. (b) Anteroposterior projection (next systole). The subaortic infundibulum (SAI) is opacified. The two infundibula are separated by a large tear-shaped crista: the conus septum (CS). The subpulmonary infundibulum (SPI) presents a very short stenosis looking like a filling defect $(\rightarrow)$ at the site of the valve-like structure found at necropsy (see Fig. 2). There is some stenosis at the bifurcation of the pulmonary artery and some poststenotic dilatation. The pulmonary valve $(P V)$ and the aortic valve $(A V)$ are both at the same level. 
noticed. Injection in the left ventricle showed a preferential filling of the aorta.

A diagnosis of double outlet right ventricle with sub-, supra-, and valvar pulmonary stenosis and L-malposition of the great arteries was made. It was suspected from left ventricular angiocardiography that the ventricular septal defect would be underneath the aortic infundibulum and that this kind of defect could be corrected in the same way as a tetralogy of Fallot. The only problem was access to the posterior pulmonary artery to relieve the stenosis.

Through a transverse incision in the right ventricle, a Dacron patch was sutured over the ventricular septal defect and over the origin of the subaortic infundibulum to direct the left ventricular flow to the aorta. An important part of the conus septum, obstructing the subpulmonary outflow, was resected and the pulmonary valve was dilated, so that the surgeon could pass his finger easily into the pulmonary artery. The girl did well initially but soon developed a low output syndrome and died suddenly on the seventh postoperative day.

At necropsy the heart was enlarged in toto with an apex formed by both ventricles. The right-sided ventricle was large and hypertrophic. It had a tricuspid AV valve and conspicuous trabeculation of the septum so that it $C$ could be identified as a morphological right ventricle. $\overrightarrow{\vec{A}}$ The parietal band was large, building a double conus divided by a huge muscular structure: the conus septum. This septum had an oblique course from left-dorsal to right-ventral, where the upper portion of the ventricular septum presented a more sagittal direction. Thus, the conus septum lay to the right of the ventricular septum, leaving a triangular septal defect between both septa. This 'ventricular septal defect' was large and joined the anterior cusp of the tricuspid valve at the commissure between the anterior and septal cusps; it was located under the subaortic infundibulum (Fig. 2a). The aorta arose from the left anterior conus. The aortic valve leaflets were at the same horizontal level as the pulmonary valve but more anterior and to the left, the noncoronary cusp being located to the left. The left coronary artery was normal but the right was hypoplastic, the origin being pin-pointed. The pulmonary artery arose from the right posterior conus and had three thickened and calcified cusps; the commissures were more or less fused. Three other semilunar fibrotic structures were found (Fig. 2b) I cm under these valve cusps, in the middle of the subpulmonary conus where this infundibulum

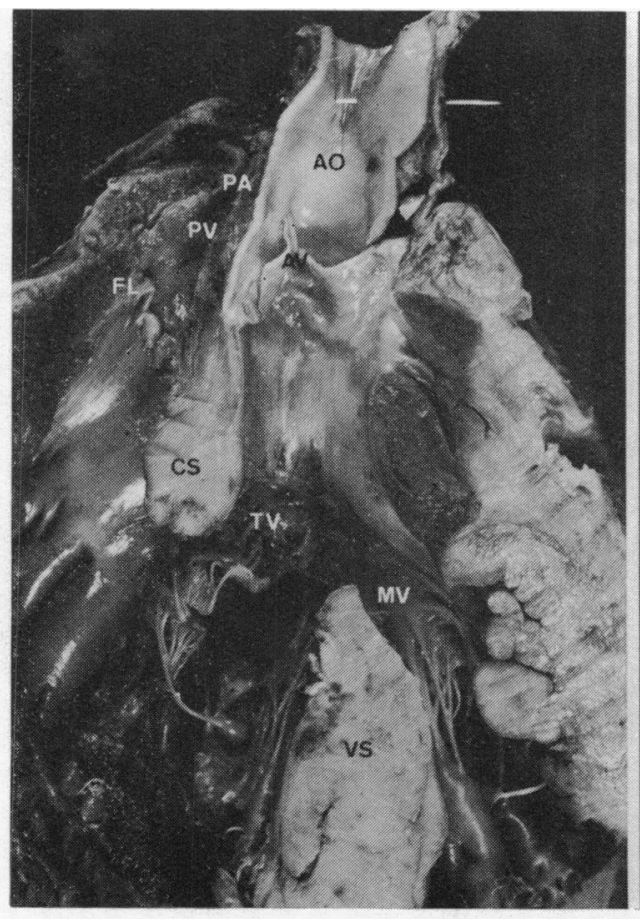

$a$

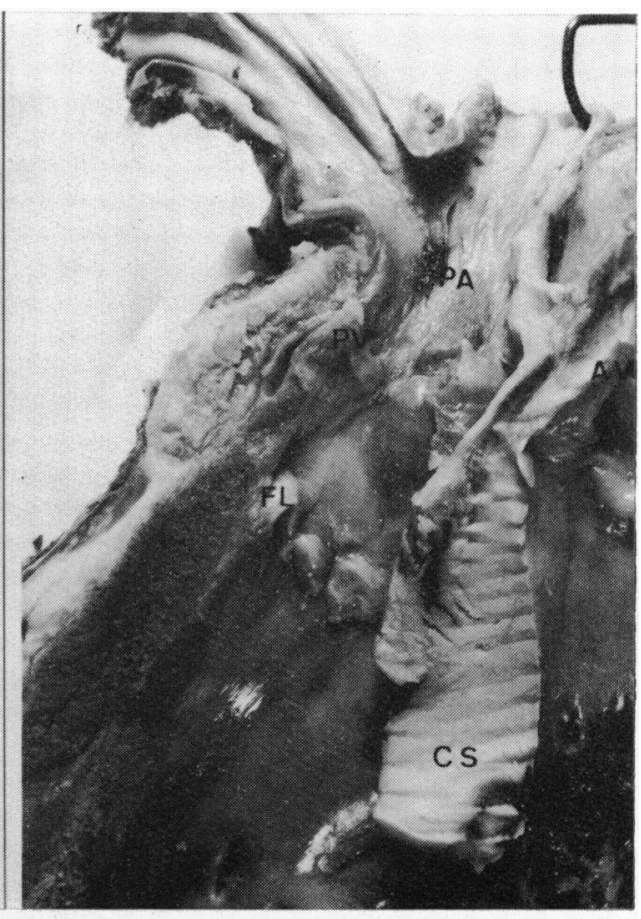

$b$

FIG. 2 Frontal section through both ventricles and both great arteries. (a) General view. (b) Detail of the subpulmonary infundibulum with the fibrotic leaflets $(F L)$ I cm below the stenotic pulmonary valve. $A O$, aorta; $P A$, pulmonary artery; $A V$, aortic valve; $P V$, pulmonary valve; $C S$, conus septum; VS, ventricular septum; $T V$, tricuspid valve; $M V$, mitral valve. 
was very narrowed. These closely resembled semilunar valves turned upside down, with their pockets looking upstream. Their edges were very thin and their commissures corresponded exactly with the commissures of the true pulmonary valve above. The atria were normal except for a posterior atrial septal defect that had been closed with one single stitch. The only outlet from the left ventricle was through the ventricular septal defect and there was no fibrous continuity between the anterior leaflet of the mitral valve and either of the semilunar valves.

\section{Discussion}

This type of double outlet right ventricle, with pulmonary stenosis, ventricular D-loop, and L-malposition of the great arteries in a situs solitus individual, cannot be differentiated clinically from tetralogy of Fallot with a right aortic arch. The angiocardiogram, however, shows clearly that both great arteries originate from a right-sided morphological right ventricle. The double conus is proved by the fact that both semilunar valves are at the same horizontal level and well separated from the mitral and tricuspid valves. Stereo or biplane angiocardiography shows the left anterior position of the aorta and the right posterior of the pulmonary artery, which confirms the L-malposition of the great arteries. The injection into the left ventricle shows clearly the preferential flow from this ventricle to the aorta and therefore the subaortic position of the ventricular septal defect.

The nature of the valve-like structure found in the middle of the subpulmonary infundibulum is not clear. The fact that the pockets are looking upstream makes it unlikely ontogenetically that they are valves, though phylogenetically this is not impossible because sharks have several columns of semilunar valves (R. van Praagh, 1972, personal communication). The fact that there are three pockets completely encircling the subpulmonary infundibulum and that the commissures between them are exactly in line with the commissures of the pulmonary valve makes it unlikely that they are systolic pockets of Zahn and Schmincke - jet lesions of the endocardium seen on the surface of the hypertrophic septum in aortic stenosis (Saphir, 1930). Though old fibrous structures have been found in the right ventricular tract in cases of corrected transposition, such a subpulmonary valve-like structure has not previously been described. The subpulmonary fibrous collar (Edwards et al., 1965) and the accessory valvular tissue (Levy et al., 1963), described in several cases of corrected transposition, are located just above the ventricular septal defect at the junction of the pulmonary and venous atrioventricular valves. In addition, these 'collars' were more like diaphragms and did not have three separated pockets.

These fibrous structures are difficult to diagnose preoperatively and may easily be overlooked during operation, as they were in this case. It may be that their removal would have further improved the pulmonary blood flow.

\section{References}

Edwards, J. E., Carey, L. S., Neufeld, H. N., and Lester, R. G. (1965). Congenital Heart Disease. Saunders, Philadelphia and London.

Hallerman, F. J., Kincaid, O. W., Ritter, D. G., Ongley, P. A., and Titus, J. L. (1970). Angiocardiographic and anatomic findings in origin of both great arteries from the right ventricle. American fournal of Roentgenology, 109, 51 .

Levy, M. J., Lillehei, C. W., Elliott, L. P., Carey, L. S., Adams, P., Jr., and Edwards, J. E. (1963). Accessory valvular tissue causing subpulmonary stenosis in corrected transposition of the great vessels. Circulation, 27, 494.

Paul, M. H., Van Praagh, S., and Van Praagh, R. (I968). Transposition of the great arteries. In Paediatric Cardiology, p. 576. Ed. by Hamish Watson. Loyd-Luke, London.

Saphir, O. (1930). Endocardial pockets. American fournal of Pathology, 6, 733.

Van Praagh, R., Perez-Trevino, C., Reynolds, J. L., Moes, C. A. F., and Keith, J. D. (1973). Double outlet right ventricle: new type with D-loop and L-malposition of the great arteries. To be published.

Requests for reprints to Dr. A. Blancquaert, Department of Pediatrics, University of Gent, Gent, Belgium. 